\title{
Vertebral hemangioma coincident with metastasis of colon adenocarcinoma
}

\author{
Krzysztof Zapałowicz, MD,' Grażyna Bierzyńska-Macyszyn, MD,2 Bartłomiej Stasiów, MD, ${ }^{3}$ \\ Aleksandra Krzan, Dr, ${ }^{4}$ Beata Wierzycka, BSN, ${ }^{1}$ and Anna Kopycka, BSN ${ }^{1}$
}

\begin{abstract}
Departments of ${ }^{1}$ Neurosurgery and ${ }^{4}$ Neurology and ${ }^{3}$ The Unit of Diagnostic Imaging, Independent Public Clinical Hospital No. 7 of the Medical University of Silesia in Katowice, Professor Leszek Giec Upper Silesian Medical Centre; and ${ }^{2}$ Histopathological Diagnostic Laboratory, Department of Pathology, Medical University of Silesia, Katowice, Poland
\end{abstract}

\begin{abstract}
The authors report on colon cancer metastasis to the L-3 vertebra, which had been previously found to be involved by an asymptomatic hemangioma. A 61-year-old female patient was admitted after onset of lumbar axial pain and weakness of the right quadriceps muscle. Her medical history included colon cancer that had been diagnosed 3 years earlier and was treated via a right hemicolectomy followed by chemotherapy. Presurgical imaging revealed an asymptomatic hemangioma in the L-3 vertebral body. Computed tomography and MRI of the spine were performed after admission and revealed a hemangioma in the L-3 vertebral body as well as a soft-tissue mass protruding from the L-3 vertebral body to the spinal canal. Treatment consisted of vertebroplasty of the hemangioma, left L-3 hemilaminectomy, and removal of the pathological mass from the spinal canal and the L-3 vertebral body. Histopathological examination revealed the presence of colon cancer metastasis and a hemangioma in the same vertebra.
\end{abstract}

http://thejns.org/doi/abs/10.3171/2015.6.SPINE141205

KEY WORDS vertebral hemangioma; colon cancer; metastasis; lumbar spine; surgical treatment; oncology

$\mathrm{V}$ ERTEBRAL hemangioma $(\mathrm{VH})$ is the most common benign vascular lesion of the spine and is present in $10 \%-12 \%$ of the population. ${ }^{6,8,15}$ The spine is also the most common site of skeletal metastases. ${ }^{6,20}$ The colon is the site of primary malignancy in $10 \%$ of patients with spinal metastases..$^{20}$ In this study, we report on colon cancer metastasis to the L-3 vertebral body (VB) that had been previously found to be involved by an asymptomatic vertebral hemangioma. Computed tomography scans and MR images revealed that the metastasis mimicked extravertebral proliferation of the hemangioma. The diagnosis was clarified by histopathological examination.

\section{Case Report}

A 61-year-old woman was admitted to the hospital in December 2013 after an onset of sharp lumbar pain radiating to her thighs associated with weakness of the right thigh. In 2009, she had undergone a right hemicolectomy followed by chemotherapy for colon cancer. Presurgical imaging had revealed an asymptomatic hemangioma in the entire L-3 VB; no treatment had been considered for this lesion. In 2013, 2 metastases were removed from the right lung. On examination, the patient demonstrated paresis of the right quadriceps muscle (3/5), the right knee reflex was diminished, the left straight leg raise test was positive, and her pain intensity was scored as 10/10 on the visual analog scale. The patient's ability to walk was limited; she could walk a few steps at a time with the assistance of an elbow crutch. On admission to the hospital, CT scans revealed that the hemangioma involved the entire VB and left pedicle of the L-3 vertebra; in addition, the posterior vertebral wall was disrupted. A soft-tissue mass was present in the posterior part of the L-3 VB. This mass occupied the anterior third of the vertebral canal and extended toward the left L3-4 intervertebral foramen (Fig. 1). Lumbar MR imaging revealed hyperintense signal of the L-3 VB on T1-weighted and T2-weighted images, which was indicative of a vertebral cavernous hemangioma. The mass 

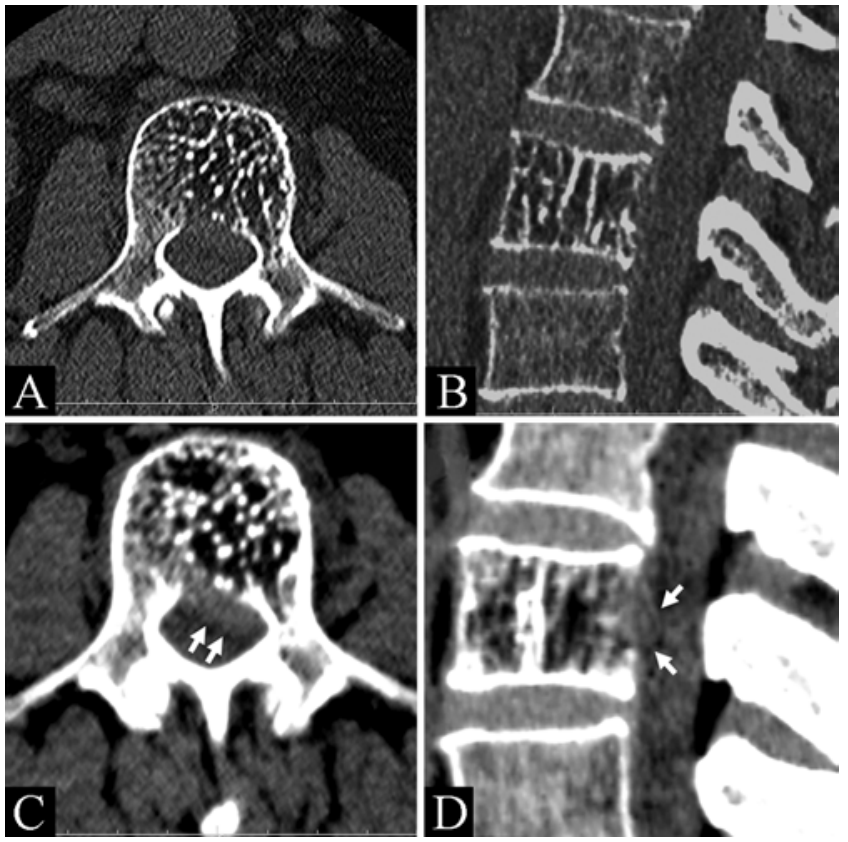

FIG. 1. CT images of the $L-3$ vertebra. Axial scan (A) and sagittal reconstruction (B) showing a polka-dot pattern and vertical striations typical for vertebral hemangioma, present in the entire VB and left pedicle. The soft-tissue mass (arrows) is present in the posterior part of the VB, in the disrupted posterior vertebral wall, and in the anterior part of the spinal canal (C and D).

in the posterior part of the L-3 VB and in the adjacent spinal canal exhibited a different signal; it was hypointense on T1-weighted images and slightly hypointense on T2weighted images and isointense on STIR sequences with intensive enhancement after gadolinium injection (Fig. 2). Given the patient's medical history, extravertebral proliferation of the $\mathrm{VH}$ or metastasis was suspected. The patient consented to undergo open surgery focused on removal of the mass from the spinal canal. After a midline incision, the retractor was placed over the left lamina of the L-3 vertebra. Vertebroplasty of the VH was performed via a left transpedicular approach to reduce possible bleeding. The left lamina of the L-3 vertebra was then removed and the dural sac was displaced medially, revealing a gray mass covered by large veins. The mass was removed from the spinal canal and curetted from the posterior part of the L-3 VB with minimal bleeding (Fig. 3). During the postoperative course, the patient's pain alleviated and her motor deficit regressed to $4 / 5$. Two months after the surgery, metastases to the pelvis were diagnosed. On pathological examination, the removed tissue samples were dense with palpable bony elements. Microscopic examination found connective tissue and bony trabeculae as well as numerous vessels of different diameters filled with red particles and clotted protein masses and focal thrombosis; these structures were intermixed with atypical tubules of metastatic colon adenocarcinoma (Fig. 4). The aforementioned findings confirmed the presence of colon cancer metastasis and hemangioma in the same vertebra.

\section{Discussion}

Clinically, vertebral hemangiomas (VHs) are classified as asymptomatic, painful (with pain as the sole symptom), and compressive (aggressive), with signs of myelopathy or nerve root compression. ${ }^{8,14,15}$ Compression is secondary to extradural extension of the $\mathrm{VH}$ or cortical bulging; rarely is it caused by hemorrhage in the vertebral canal
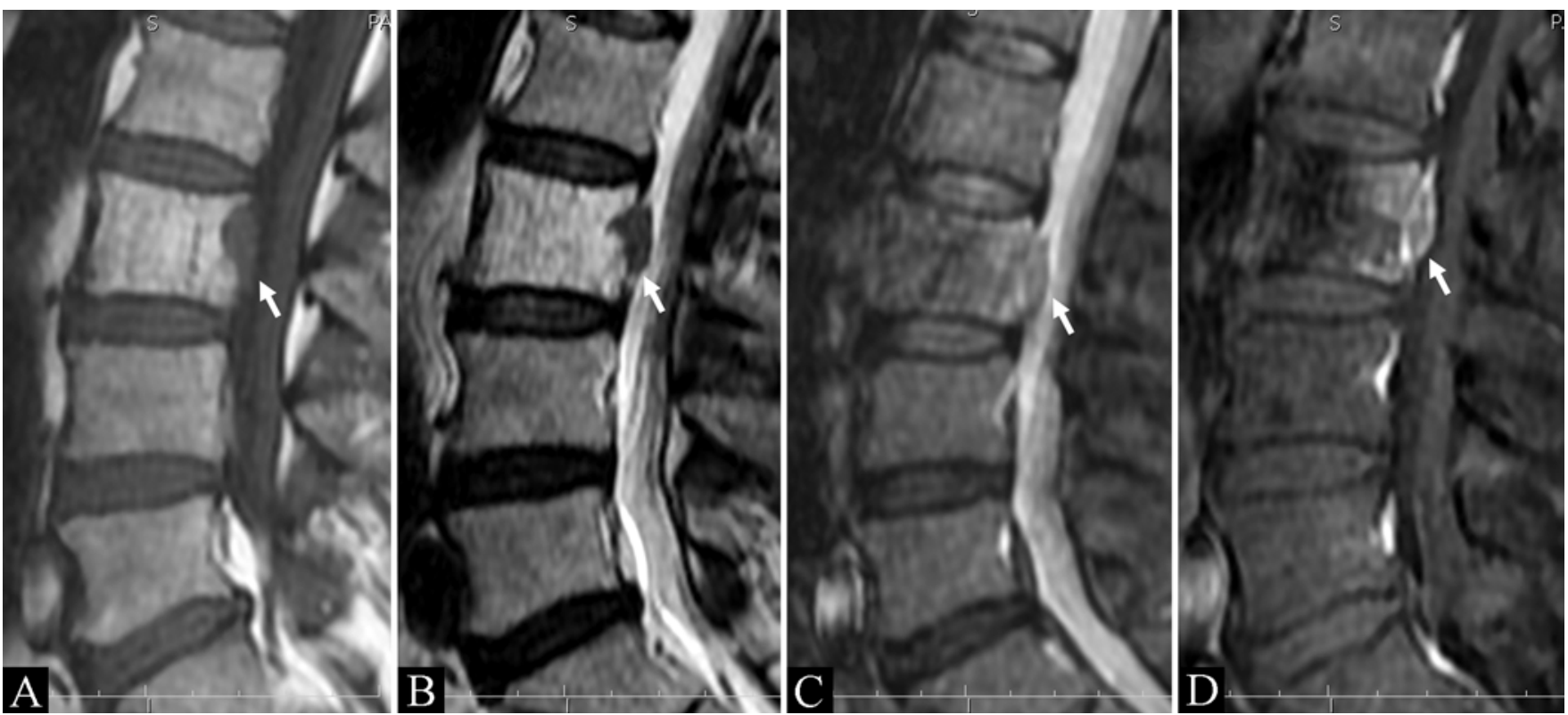

FIG. 2. MR images of the L-3 vertebra. A hyperintense signal of the VB is shown on T1-weighted (A) and T2-weighted (B) images. The well-limited mass in the posterior part of the L-3 VB (arrows) and in the adjacent spinal canal is hypointense to the normal marrow on the T1-weighted image (A), slightly hypointense on the T2-weighted image (B), and isointense on the STIR sequence (C), with intensive enhancement after gadolinium injection (D). 

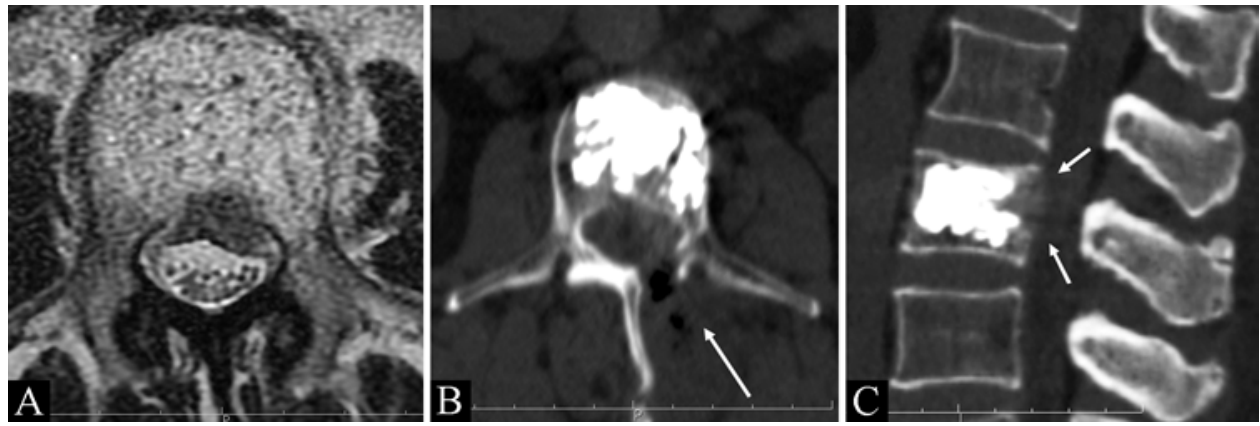

FIG. 3. Preoperative axial T2-weighted MR image showing the largest degree of spinal canal compromise, estimated to be between $25 \%$ and $30 \%$ (A). Axial CT scan (B) and sagittal reconstruction (C) obtained on the 7 th postoperative day, showing acrylic cement filling the hemangioma. The spinal canal is opened via a left hemilaminectomy (arrow in B) and is freed from any extradural mass (arrows in C).

or vertebral fracture. ${ }^{1,6,8,9,15}$ There are reports on the rapid onset of signs of $\mathrm{VH}$ in the third trimester of pregnancy, suggesting that growth of the hemangioma can be stimulated by elevated secretion of estrogen with contribution of increased intraabdominal pressure. .,8, $, 15,16^{2}$ To the best of our knowledge, spontaneous malignant transformation of VHs has not been reported. 2,3,8 According to criteria highlighted by Laredo and colleagues, aggressive VHs most frequently affect vertebrae from T-3 to T-9 and involve the entire VB, pedicles, and vertebral arch. Moreover, they exhibit irregular trabeculation, expanded and poorly defined cortex, disrupted cortex, and swelling of the paravertebral soft tissue. ${ }^{14,15}$ On MRI, aggressive VHs show low signal intensity on T1-weighted images and high signal intensity on T2-weighted images because of the low fat content and rich vascular component. ${ }^{8,14,15}$ Spinal metastatic disease is associated with pain or neurological deficit in about $10 \%$ of cancer patients, mainly by mechanical compression of the spinal cord or roots. ${ }^{4,11}$ Compression is a consequence of tumor proliferation to the spinal canal, vertebral bulging, or fracture. ${ }^{11}$ Other postulated mechanisms of neurological deficit are ischemia or edema of the spinal cord. ${ }^{11}$

Colorectal cancer is the third most common solid tumor in the adult population after lung, and breast and prostate cancers combined..$^{18,19}$ In 1995, Sioutos et al. estimated that the colon was the primary malignancy site in $10 \%$ of patients with spinal metastases. ${ }^{20}$ In the described case, the VH diagnosed in 2009 was suspected to proliferate toward the spinal canal. This suspicion was supported by $\mathrm{CT}$ and MR imaging that revealed the following radiological features of aggressiveness: involvement of the entire $\mathrm{VB}$ and one pedicle, disruption of the posterior vertebral cortex, and the soft-tissue mass in the spinal canal close to the cortical disruption. ${ }^{15}$ However, the radiological appearance of spinal metastasis may be similar, making the proper diagnosis difficult. ${ }^{2,16}$ We chose surgical removal of the compressive lesion from the spinal canal. The major complication associated with surgical removal of VHs is extensive blood loss. ${ }^{1,2,-10}$ To reduce assumed bleeding, despite being the origin of the compressive lesion, vertebroplasty of the affected VB was performed before opening the spinal canal. Vertebroplasty consisted of an injection of acrylic bone cement into the affected VB. Cement em- bolizes the vessels and fills spaces between bone trabeculae, thus reducing blood loss. ${ }^{5,6,12}$ After vertebroplasty, the remaining surgical procedure was performed with minimal blood loss and resulted in pain relief with regression of paresis. Histopathological examination revealed an unusual coincidence of a hemangioma and metastatic colon carcinoma in the same lumbar vertebra. Colorectal cancer metastases spread mainly through veins and lymphatic chains to the liver and lungs, and thereafter via dissemination in the arterial circulation to different organs including the vertebral VBs. ${ }^{13,17,18}$ It is highly probable that this was the route of vertebral invasion in the presented case of a patient with history of hepatic and pulmonary metastases. However, metastatic colonization from the extradural space via the Batson venous plexus cannot be ruled out.
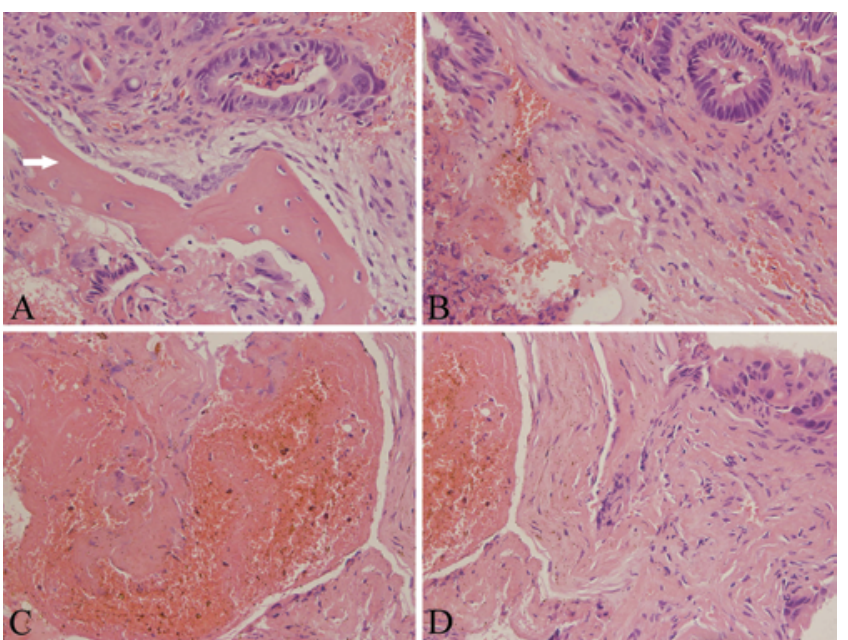

FIG. 4. Photomicrographs showing morphological features of the mass removed from the spinal canal and curetted from the L-3 VB. A: Bone trabeculae (arrow) surrounded by connective tissue and tubules of metastatic adenocarcinoma. B: The connective fibrous tissue containing cavities is lined with endothelium and filled with blood. In their proximity neoplastic tubules of metastatic adenocarcinoma are seen. C: The patterns of numerous vascular spaces filled with blood and clots. D: The pattern of blood vessel filled with clot. Connective tissue with atypical tubules of metastatic adenocarcinoma can be seen on the right side. $H \& E$, original magnification $\times 200$. 
The Batson plexus is a system of valveless veins that connect pelvic, abdominal, and thoracic veins with the venous plexus located extradurally in the spinal canal; it constitutes an alternative "direct" means of spreading metastatic cells of different origin, including colorectal cancer. . $^{13,17}$ Vertebral bodies containing a well-vascularized cancellous bone are considered to be a good environment for colonization by metastases. ${ }^{11,17}$ One may hypothesize that vertebral hemangioma composed of vascular spaces with potentially increased blood supply might provide favorable opportunity for implantation of metastatic cells.

\section{Acknowledgments}

We thank Adam Dudziński, MA, for his linguistic review of the manuscript.

\section{References}

1. Boschi V, Pogorelić Z, Gulan G, Perko Z, Grandić L, Radonić V: Management of cement vertebroplasty in the treatment of vertebral hemangioma. Scand J Surg 100:120 124,2011

2. Bremnes RM, Hauge HN, Sagsveen R: Radiotherapy in the treatment of symptomatic vertebral hemangiomas: technical case report. Neurosurgery 39:1054-1058, 1996

3. Chen HI, Heuer GG, Zaghloul K, Simon SL, Weigele JB, Grady MS: Lumbar vertebral hemangioma presenting with the acute onset of neurological symptoms. Case report. J Neurosurg Spine 7:80-85, 2007

4. Choi D, Crockard A, Bunger C, Harms J, Kawahara N, Mazel C, et al: Review of metastatic spine tumour classification and indications for surgery: the consensus statement of the Global Spine Tumour Study Group. Eur Spine J 19:215222, 2010

5. Cotten A, Boutry N, Cortet B, Assaker R, Demondion X, Leblond D, et al: Percutaneous vertebroplasty: state of the art. Radiographics 18:311-323, 1998

6. Deramond H, Chiras J, Cotten A: Tumors, in Mathis JM, Deramond H, Belkoff SM (eds): Percutaneous Vertebroplasty and Kyphoplasty, ed 2. New York: Springer, 2006, pp 157-184

7. Doppman JL, Oldfield EH, Heiss JD: Symptomatic vertebral hemangiomas: treatment by means of direct intralesional injection of ethanol. Radiology 214:341-348, 2000

8. Fox MW, Onofrio BM: The natural history and management of symptomatic and asymptomatic vertebral hemangiomas. J Neurosurg 78:36-45, 1993

9. Hadjipavlou A, Tosounidis T, Gaitanis I, Kakavelakis K, Katonis P: Balloon kyphoplasty as a single or as an adjunct procedure for the management of symptomatic vertebral haemangiomas. J Bone Joint Surg Br 89:495-502, 2007

10. Hao J, Hu Z: Percutaneous cement vertebroplasty in the treatment of symptomatic vertebral hemangiomas. Pain Physician 15:43-49, 2012
11. Heary RF, Bono CM: Metastatic spinal tumors. Neurosurg Focus 11(6): 1,2001

12. Ide C, Gangi A, Rimmelin A, Beaujeux R, Maitrot D, Buchheit F, et al: Vertebral haemangiomas with spinal cord compression: the place of preoperative percutaneous vertebroplasty with methyl methacrylate. Neuroradiology 38:585-589, 1996

13. Katoh M, Unakami M, Hara M, Fukuchi S: Bone metastasis from colorectal cancer in autopsy cases. J Gastroenterol 30:615-618, 1995

14. Laredo JD, Assouline E, Gelbert F, Wybier M, Merland JJ, Tubiana JM: Vertebral hemangiomas: fat content as a sign of aggressiveness. Radiology 177:467-472, 1990

15. Laredo JD, Reizine D, Bard M, Merland JJ: Vertebral hemangiomas: radiologic evaluation. Radiology 161:183-189, 1986

16. Lee JH, Park CW, Chung DH, Kim WK: A case of lumbar metastasis of choriocarcinoma masquerading as an extraosseous extension of vertebral hemangioma. J Korean Neurosurg Soc 47:143-147, 2010

17. Maccauro G, Spinelli MS, Mauro S, Perisano C, Graci C, Rosa MA: Physiopathology of spine metastasis. Int J Surg Oncol 2011:107969, 2011

18. Roth ES, Fetzer DT, Barron BJ, Joseph UA, Gayed IW, Wan DQ: Does colon cancer ever metastasize to bone first? A temporal analysis of colorectal cancer progression. BMC Cancer 9:274, 2009

19. Santini D, Tampellini M, Vincenzi B, Ibrahim T, Ortega C, Virzi V, et al: Natural history of bone metastasis in colorectal cancer: final results of a large Italian bone metastases study. Ann Oncol 23:2072-2077, 2012

20. Sioutos PJ, Arbit E, Meshulam CF, Galicich JH: Spinal metastases from solid tumors. Analysis of factors affecting survival. Cancer 76:1453-1459, 1995

\section{Disclosures}

The authors report no conflict of interest concerning the materials or methods used in this study or the findings specified in this paper.

\section{Author Contributions}

Conception and design: Zapałowicz. Acquisition of data: all authors. Analysis and interpretation of data: Zapałowicz, Bierzyńska-Macyszyn, Stasiów. Drafting the article: Zapałowicz. Critically revising the article: Zapałowicz. Approved the final version of the manuscript on behalf of all authors: Zapałowicz.

\section{Correspondence}

Krzysztof Zapałowicz, Department of Neurosurgery, Independent Public Clinical Hospital No. 7 of the Medical University of Silesia in Katowice, Professor Leszek Giec Upper Silesian Medical Centre, 45/47 Ziołowa St., Katowice 40-635, Poland. email: krzysztofzapalowicz1@wp.pl. 